Published in final edited form as:

Head Neck. 2014 April ; 36(4): 511-516. doi:10.1002/hed.23318.

\title{
Human papillomavirus and Epstein-Barr virus in nasopharyngeal carcinoma in a low-incidence population
}

\author{
Snjezana Dogan, MD¹, Matthew L. Hedberg, BS ${ }^{2}$, Robert L. Ferris, MD, PhD ${ }^{3}$, Tanya J. Rath, \\ $\mathbf{M D}^{4}$, Adel M. Assaad, $\mathbf{M D}^{5}$, and Simion I. Chiosea, $\mathbf{M D}^{6,{ }^{*}}$ \\ ${ }^{1}$ Department of Pathology, Memorial Sloan-Kettering Cancer Center, New York, New York \\ ${ }^{2}$ University of Pittsburgh-Carnegie Mellon University, Pittsburgh, Pennsylvania \\ ${ }^{3}$ Department of Otolaryngology/Head and Neck Surgery, University of Pittsburgh Medical Center, \\ Pittsburgh, Pennsylvania \\ ${ }^{4}$ Department of Radiology, University of Pittsburgh Medical Center, Pittsburgh, Pennsylvania \\ ${ }^{5}$ Department of Pathology, Virginia Mason Medical Center, Seattle, Washington \\ ${ }^{6}$ Department of Pathology, University of Pittsburgh Medical Center, Pittsburgh, Pennsylvania
}

\begin{abstract}
Background-The significance of human papillomavirus (HPV) in nasopharyngeal carcinomas (NPCs) in a low-incidence population remains unknown.

Methods-Samples from 90 patients with NPC (years, 1957-2012) were analyzed for EpsteinBarr virus (EBV). Clinical data, EBV, HPV, and p16 status were correlated with overall survival (OS; 63 cases; years, 1981-2012).

Results-Of $9 \mathrm{HPV}$-positive cases, 3 extended from extra-nasopharyngeal sites. Nasopharyngeal origin was confirmed in 6 cases. HPV-positive NPC had OS similar to EBV-positive NPC (85 vs 141 months; $p>.05$ ). The OS of patients with EBV/HPV-negative NPC was worse (34 months; $p$ $=.004)$. Nonkeratinizing histology was associated with better outcome than keratinizing (115 vs 25 months; $p=.001)$. Over the last several decades, the proportion of keratinizing NPC decreased from $34.5 \%$ to $14.3 \%(p=.026)$.
\end{abstract}

Conclusion-The etiologic role of HPV in NPC is confirmed. The favorable prognostic significance of HPV positivity is similar to that of EBV positivity.

\section{Keywords}

nasopharyngeal carcinoma; human papillomavirus; Epstein-Barr virus

*Corresponding author: S. I. Chiosea, Presbyterian University Hospital, A610.3, 200 Lothrop St., Pittsburgh, PA 15213. chioseasi@upmc.edu.

This work was presented at the United States and Canadian Academy of Pathology meeting, March, 2012. 


\section{INTRODUCTION}

Epstein-Barr virus (EBV) has been long recognized as an etiologic agent of nasopharyngeal carcinoma (NPC), a tumor with distinct racial and geographic distribution. ${ }^{1,2}$ However, over the last decade, several reports identified human papillomavirus (HPV) in NPC. ${ }^{3-7}$ Few of the prior studies focused on low-incidence population and used combination of p16 immunohistochemistry (IHC) and HPV in situ hybridization (ISH) to distinguish pathogenically relevant HPV infection from incidental. ${ }^{3,5}$ Furthermore, because an unequivocal majority of HPV-associated squamous cell carcinomas (SCCs) in the head and neck arise in the oropharynx, ${ }^{8,9}$ it was noted that most if not all HPV-positive "NPC" might actually represent extension of oropharyngeal SCC into the nasopharynx. ${ }^{5}$

The purpose of this study was to estimate the prevalence of HPV-positive NPC by complementing p16 IHC and HPV ISH studies with clinical and imaging data. Once the nasopharyngeal origin of HPV-positive carcinoma was confirmed, for the first time, overall survival (OS) of patients with HPV-positive NPC was directly compared to that of EBVpositive and HPV/EBV-negative NPC and the prognostic significance of histology (ie, keratinizing vs nonkeratinizing), age, sex, and staging was reassessed.

In addition to viral etiology, smoking has been implicated in the carcinogenesis of NPC. ${ }^{10}$ Importantly, in low-incidence population, smoking was associated with keratinizing rather than nonkeratinizing NPC. Given the reduction in cigarette smoking in the United States over the last several decades, ${ }^{11}$ we hypothesized that decline in tobacco exposure would be associated with lower prevalence of keratinizing NPC. To indirectly test this hypothesis and to describe the morphologic evolution of NPC, demographic, morphologic features, and EBV status were compared between cases diagnosed from 1956 to 1977 and those from 1981 to 2012.

\section{PATIENTS AND METHODS}

\section{Patients}

Patients from 2 time periods were studied. From 1981 to March of 2012, 67 patients with NPC were identified, including 58 cases from the University of Pittsburgh Medical Center, Pittsburgh, PA and 9 cases from Virginia Mason Medical Center, Seattle, Washington (referred to as group 1). Clinical records, including operative reports and radiographic imaging, were reviewed. The clinical stage was determined according to the American Joint Committee on Cancer Staging Manual, 7th edition. ${ }^{12}$ NPC were histologically classified as keratinizing (World Health Organization [WHO] type I) and nonkeratinizing NPC (WHO type II). ${ }^{13}$ Because the clinical significance of differentiated and undifferentiated nonkeratinizing histology is similar, further subtyping of WHO type II was not performed. The nasopharyngeal origin of carcinoma was accepted only if clinical examination (including intra-operative findings) and radiographic data showed the bulk of the tumor in the nasopharynx without any evidence of oropharyngeal or sinonasal extension. The imaging studies of 4 patients with HPV-positive NPC were reviewed by a radiologist (author T.J.R.). In other cases, reports of imaging studies and/or actual imaging studies were reviewed by authors R.L.F. and S.I.C. 
The pathology archives of the University of Pittsburgh Medical Center were also searched for NPC diagnosed from 1956 to 1977, as previously described. ${ }^{14}$ Thirty-four cases of NPC were identified (referred to as group 2). Hematoxylin-eosin stained slides were available for 29 cases. Formalin-fixed paraffin-embedded tissue blocks were available for 27 of 29 cases. All medical records from that time period had been destroyed; only minimal clinical information provided on pathology reports was available. Unfortunately, patients' ethnicity, smoking history, or clinical follow-up could not be determined for group 2.

This work was approved by the University of Pittsburgh Institutional Review Board (IRB \#PRO10100585) and Total Quality Council.

\section{Epstein-Barr virus, human papillomavirus detection, and p16 immunohistochemistry}

EBV detection was performed by ISH using probes targeting EBV encoded early RNA (EBER; catalog \#780-2842; Ventana Discovery XT, Tucson, AZ). All cases tested for EBER were also tested for the integrity of total RNA using RNA-positive control probe. One case from group 2 showed inadequate total RNA preservation and in 3 cases no carcinoma was identified on deeper tissue section used for EBV ISH.

All EBV-negative cases and 8 EBV-positive cases, from 1981 to 2012, were tested by HPV ISH and p16 IHC. The HPV probe cocktail included a wide spectrum of HPV strains including 6, 11, 16, 18, 31, 33, 35, 39, 45, 51, and 52 (Y1404; Dako, Carpinteria, CA) and cases with dot-like/punctate nuclear signal were considered positive. ${ }^{15}$ Group 2 patients were not tested for HPV/p16, as correlation with clinical and imaging data to confirm nasopharyngeal site would be impossible.

p16 IHC (G175-405; BD Pharmingen, San Diego, CA) was performed according to the manufacture's protocol. Cases were considered positive if more than $70 \%$ of tumor cells showed diffuse strong cytoplasmic and nuclear staining. ${ }^{16}$ It was previously reported that p16 IHC is negative in EBV-positive NPC. ${ }^{17,18}$

\section{Statistical analysis}

The primary endpoint was OS (mean estimated), measured from the date of diagnosis to the date of death from any cause. Alive patients were censored on the date of last follow-up. Kaplan-Meier method and log-rank test were used to examine survival differences among groups. Chi-square or Fisher exact tests were used to examine associations between categorical variables. The $t$ test was used to examine associations between continuous variables. A $p$ value of .05 or less was considered significant. All statistical analyses were done using SPSS Statistics 20 software (IBM, Armonk, NY).

\section{RESULTS}

\section{Confirming nasopharyngeal origin of carcinoma in patients diagnosed from 1981 to 2012}

To assure the nasopharyngeal site of carcinoma, clinical data and radiographic findings of all 67 patients diagnosed from 1981 to March 2012 were reviewed. History of tonsillectomy was recorded. Four cases were shown to be of non-nasopharyngeal origin and were excluded from further analysis. In 1 case, according to all clinical data, SCC was actually involving 
the hypopharynx, rather than the nasopharynx (as it was erroneously indicated on the pathology requisition form). In 2 patients with HPV-positive and p16-positive SCC, the bulk of the disease was in the oropharynx.

The fourth excluded case was that of a 50-year-old man (never smoker, no history of alcohol abuse) who presented with VIth cranial nerve palsy. Although the diagnostic biopsy was obtained from the nasopharynx, imaging studies showed that the bulk of the $5 \mathrm{~cm}$ mass was in the ethmoid sinus. Histologically, nonkeratinizing HPV-positive SCC (cylindrical) was identified. ${ }^{19}$

\section{Patients with confirmed human papillomavirus-positive nasopharyngeal carcinoma}

A detailed description of patients with HPV/p16-positive NPC is presented in Table 1 (all p16 IHC-positive cases were also HPV ISH-positive). All patients were white. Five patients were men and 1 patient was a woman (case \#5). Review of clinical and radiographic data showed no evidence of oropharyngeal or sinonasal involvement. One patient (case \#2) had a tonsillectomy and base of tongue biopsy ipsilateral to nasopharyngeal and neck masses; the tonsillectomy and base of tongue biopsy were free of carcinoma. Five cases were nonkeratinizing, whereas case \#6 revealed keratinizing histology. EBER ISH was negative in all 6 cases.

\section{Overall characterization of patients with nasopharyngeal carcinoma}

Clinical and pathologic data for patients in groups 1 and 2 are compared in Table 2. When groups 1 and 2 were compared, patients' mean age was similar -53 years (range, 21-85 years; age of 1 man in group 1 was unknown). NPC affected predominantly men, both in group 1 (44 of 63) and in group 2 (25 of 29). Histologically, there was a decrease in number of keratinizing NPC: from $34.5 \%$ of patients (10 of 29) in group 2 to $14.3 \%$ of patients ( 9 of 63 ) in group $1(p=.026)$. Combined analysis of 92 patients revealed that keratinizing NPC was seen in patients who were on average 7 years older than patients with nonkeratinizing NPC (59 vs 52 years; $p=.04$ ). EBV-positive NPCs were predominant in both groups (15 of $29 ; 52 \% ; 1956-1977$ vs 38 of $63 ; 60 \% ; 1981-2012)$. Eight EBV-positive NPCs were tested for HPV ISH and p16 IHC and were negative.

In group 1, presenting symptoms were known for 39 patients. Neck mass was the most common initial complaint ( 19 of $39 ; 48.7 \%$ ), followed by headache ( 6 of $39 ; 15.4 \%$ ), epistaxis ( 5 of $39 ; 12.8 \%$ ), and hearing loss ( 5 of $39 ; 12.8 \%$ ). Adequate history of alcohol intake was available for 31 patients and history of alcohol abuse was noted in 5 patients. Detailed description of patients in group 1 is presented in Table 3.

\section{Overall survival analysis - role of sex, histology, and Epstein-Barr virus/human papillomavirus status}

The median follow-up was 38 months. Male patients had significantly better OS than women ( $p=.008$; Figure 1A). The OS of women was 40.4 months ( $95 \%$ confidence interval [CI], 25-56 months), whereas for men it was 130 months (95\% CI, 67-193 months).

Nonkeratinizing histology of NPC was associated with better outcome than keratinizing ( $p$ $=.001$; Figure 1B). The OS of patients with keratinizing NPC was 25 months (95\% CI, 8-42 
months), whereas for patients with nonkeratinizing NPC, it was 115 months (95\% CI, 62169 months). In subgroup analysis, the adverse prognostic significance of keratinizing morphology was maintained both among women $(p<.001)$ and men $(p=.003)$.

When HPV status of EBV-negative NPCs was not accounted for, the OS of patients with EBV-positive NPC was significantly longer than of those with EBV-negative NPC ( $p=$. 013; Figure 1C): the OS of patients with EBV-positive NPC was 141 months (95\% CI, 69212 months), whereas for patients with EBV-negative NPC, it was 46 months (95\% CI, 2665 months).

The OS of patients with HPV-positive NPC (85 months; 95\% CI, 31-139 months) was not significantly different from that of EBV-positive NPC (141 months; 95\% CI, 69-212 months). However, the OS of patients with EBV-negative and HPV-negative NPC was significantly shorter (34 months; 95\% CI, 18-51 months; $p=.004$; Figure 1D). Age, TNM classification, and clinical stage were not predictive of OS.

\section{DISCUSSION}

Our analysis of NPC in a low-incidence population showed that about $40 \%$ of cases were EBV-negative. This finding and prior reports ${ }^{3-7}$ prompted us to test EBV-negative cases for HPV. Indeed, 9 of 66 cases were HPV-positive. However, 3 HPV-positive carcinomas did not seem to originate in the nasopharynx: in addition to previously reported possibility of HPV-positive oropharyngeal carcinoma extending to the nasopharynx, ${ }^{5}$ we presented a case of an HPV-positive sinonasal SCC (cylindrical) ${ }^{19}$ masquerading as NPC. Therefore, oropharyngeal or sinonasal carcinoma extending to the nasopharynx should be excluded before accepting the diagnosis of an HPV-positive NPC. We have identified 6 HPV-positive cases, representing 24\% (6 of 25) of EBV-negative NPCs.

Previously, HPV positivity in SCCs metastatic to cervical lymph node in patients with occult primary carcinoma was considered indicative of oropharyngeal source. ${ }^{20}$ Our results highlighted the need to consider the nasopharynx and sinonasal tract, as well.

In addition, for the first time, we show that HPV-positive NPCs seem to have OS similar to that of EBV-positive NPC. Others have reported favorable prognostic significance of EBVpositivity in NPC in low-incidence population (with unknown HPV status). ${ }^{21}$ Routine testing of EBV-negative NPC for HPV/p16 may help to identify a subset of NPC with worse outcome (ie, patients with EBV-negative and HPV-negative NPC).

Previously, HPV was detected in NPC with keratinizing ${ }^{22,23}$ and nonkeratinizing ${ }^{3}$ histology. Accordingly, we found 5 of 54 nonkeratinizing NPCs and 1 of 9 keratinizing NPCs to be HPV positive. HPV in NPC was previously associated with keratinizing morphology, ${ }^{23} \mathrm{a}$ known adverse factor. ${ }^{24,25}$ Given this association, we reevaluated and confirmed the adverse prognostic significance of keratinizing morphology in a cohort of patients with NPC with known EBV and HPV status. The low number of HPV-positive NPCs precluded subgroup analysis to discern the prognostic significance of histology in this subset of NPC. 
Comparison of NPC in patients from 1956 to 1977 to those from 1981 to 2012 revealed that keratinizing histology was more common in the earlier group. This finding expands on and is consistent with the predominance of keratinizing histology previously reported in a study of NPC diagnosed in the United States over 1 time period, 1985 to $1994 .{ }^{26}$ In agreement with a prior report, ${ }^{26}$ patients with keratinizing NPC were older compared to patients with nonkeratinizing NPC.

Although smoking history in our report is inadequate, the link between risk of developing keratinizing NPC and cigarette smoking was previously noted. ${ }^{10,27}$ The decrease in prevalence of keratinizing NPC is consistent with the overall decline in cigarette smoking in the United States over the last several decades. ${ }^{28}$

This study is limited by its retrospective design, incomplete demographic, smoking data (especially for group 2), low sensitivity of HPV detection methods, and lack of data on HPV subtypes. Furthermore, patients presented in this study were treated over a decades-long period. Changes in histological classification, staging, treatment protocols, and lack of HPV status in prior studies preclude adequate comparisons to other reports. Low number of patients in certain clinicopathological subgroups limited interpretation of some of our findings. For instance, women with NPC had worse outcome in this study. The significance and the reason behind this finding are unclear.

Prior studies have shown that a subset of EBV-positive NPC may also harbor HPV ( 9 of 15 cases $^{6}$ and 14 cases, all patients of Asian ethnicity ${ }^{29}$ ). These studies were performed in an ethnically different population and had advantage of more sensitive methods of HPV and EBV detection (eg, sequencing and polymerase chain reaction). In our study, $8 \mathrm{EBV}$ positive cases were p16 IHC and HPV ISH negative.

In summary, this study of NPC in a predominantly low-incidence population confirmed the existence of bona fide HPV-positive NPC. Routine testing for HPV/p16 of EBV-negative NPC may be of clinical interest, particularly in patients with occult primary carcinoma, in which HPV positivity should direct the clinician to investigate the nasopharynx and sinonasal area in addition to the oropharynx. We showed that prognostic significance of HPV positivity in NPC was similar to that of EBV positivity. The adverse prognostic impact of keratinizing histology was reassessed and confirmed in our cohort of NPC with known HPV status. Finally, morphologically, NPC evolved over the last several decades to predominantly nonkeratinizing histology.

\section{Acknowledgments}

The authors wish to thank members of the In Situ Hybridization and Developmental Laboratory of the Department of Pathology, University of Pittsburgh for excellent technical support and Robyn Roche for outstanding secretarial support.

\section{References}

1. Nonoyama M, Huang CH, Pagano JS, Klein G, Singh S. DNA of Epstein-Barr virus detected in tissue of Burkitt's lymphoma and nasopharyngeal carcinoma. Proc Natl Acad Sci U S A. 1973; 70:3265-3268. [PubMed: 4361687] 
2. Mould RF, Tai TH. Nasopharyngeal carcinoma: treatments and outcomes in the 20th century. Br J Radiol. 2002; 75:307-339. [PubMed: 12000691]

3. Maxwell JH, Kumar B, Feng FY, et al. HPV-positive/p16-positive/EBV-negative nasopharyngeal carcinoma in white North Americans. Head Neck. 2010; 32:562-567. [PubMed: 19757421]

4. Punwaney R, Brandwein MS, Zhang DY, et al. Human papillomavirus may be common within nasopharyngeal carcinoma of Caucasian Americans: investigation of Epstein-Barr virus and human papillomavirus in eastern and western nasopharyngeal carcinoma using ligation-dependent polymerase chain reaction. Head Neck. 1999; 21:21-29. [PubMed: 9890347]

5. Singhi AD, Califano J, Westra WH. High-risk human papillomavirus in nasopharyngeal carcinoma. Head Neck. 2012; 34:213-218. [PubMed: 21484924]

6. Rassekh CH, Rady PL, Arany I, et al. Combined Epstein-Barr virus and human papillomavirus infection in nasopharyngeal carcinoma. Laryngoscope. 1998; 108:362-367. [PubMed: 9504608]

7. Giannoudis A, Ergazaki M, Segas J, et al. Detection of Epstein-Barr virus and human papillomavirus in nasopharyngeal carcinoma by the polymerase chain reaction technique. Cancer Lett. 1995; 89:177-181. [PubMed: 7889526]

8. Gillison ML, Koch WM, Capone RB, et al. Evidence for a causal association between human papillomavirus and a subset of head and neck cancers. J Natl Cancer Inst. 2000; 92:709-720. [PubMed: 10793107]

9. Klussmann JP, Gültekin E, Weissenborn SJ, et al. Expression of p16 protein identifies a distinct entity of tonsillar carcinomas associated with human papillomavirus. Am J Pathol. 2003; 162:747753. [PubMed: 12598309]

10. Vaughan TL, Shapiro JA, Burt RD, et al. Nasopharyngeal cancer in a low-risk population: defining risk factors by histological type. Cancer Epidemiol Biomarkers Prev. 1996; 5:587-593. [PubMed: 8824359]

11. Sturgis EM, Cinciripini PM. Trends in head and neck cancer incidence in relation to smoking prevalence: an emerging epidemic of human papillomavirus-associated cancers? Cancer. 2007; 110:1429-1435. [PubMed: 17724670]

12. Edge, SB.; Byrd, DR.; Compton, CC.; Fritz, AG.; Greene, FL.; Trotti, A., editors. AJCC Cancer Staging Handbook: from the AJCC Cancer Staging Manual. New York: Springer; 2009.

13. Barnes, L.; Eveson, JW.; Reichart, P.; Sidransky, D. Pathology and genetics of head and neck tumours. Vol. 9. World Health Organization; 2005.

14. Smith MA, Barnes EL, Chiosea SI. Pathology archive: evaluation of integrity, regulatory compliance, and construction of searchable database from print reports. Am J Clin Pathol. 2011; 135:753-759. [PubMed: 21502430]

15. Fakhry C, Westra WH, Li S, et al. Improved survival of patients with human papillomaviruspositive head and neck squamous cell carcinoma in a prospective clinical trial. J Natl Cancer Inst. 2008; 100:261-269. [PubMed: 18270337]

16. Singhi AD, Westra WH. Comparison of human papillomavirus in situ hybridization and p16 immunohistochemistry in the detection of human papillomavirus-associated head and neck cancer based on a prospective clinical experience. Cancer. 2010; 116:2166-2173. [PubMed: 20186832]

17. Shibosawa E, Tsutsumi K, Koizuka I, Hoshikawa M, Takakuwa T. Absence of nuclear p16 from Epstein-Barr virus-associated undifferentiated nasopharyngeal carcinomas. Laryngoscope. 2000; 110:93-97. [PubMed: 10646722]

18. Mäkitie AA, MacMillan C, Ho J, et al. Loss of p16 expression has prognostic significance in human nasopharyngeal carcinoma. Clin Cancer Res. 2003; 9:2177-2184. [PubMed: 12796384]

19. El-Mofty SK, Lu DW. Prevalence of high-risk human papillomavirus DNA in nonkeratinizing (cylindrical cell) carcinoma of the sinonasal tract: a distinct clinicopathologic and molecular disease entity. Am J Surg Pathol. 2005; 29:1367-1372. [PubMed: 16160480]

20. Begum S, Gillison ML, Ansari-Lari MA, Shah K, Westra WH. Detection of human papillomavirus in cervical lymph nodes: a highly effective strategy for localizing site of tumor origin. Clin Cancer Res. 2003; 9:6469-6475. [PubMed: 14695150]

21. Terzic TT, Boricic MI, Pendjer IP, et al. Prognostic significance of clinical parameters and Epstein-Barr virus infection in non-endemic undifferentiated carcinoma of nasopharyngeal type: a Serbian report. Med Oncol. 2011; 28:1325-1330. [PubMed: 20446059] 
22. Hording U, Nielsen HW, Daugaard S, Albeck H. Human papillomavirus types 11 and 16 detected in nasopharyngeal carcinomas by the polymerase chain reaction. Laryngoscope. 1994; 104(1 Pt 1): 99-102. [PubMed: 8295466]

23. Lo EJ, Bell D, Woo JS, et al. Human papillomavirus and WHO type I nasopharyngeal carcinoma. Laryngoscope. 2010; 120:1990-1997. [PubMed: 20824783]

24. Hoppe RT, Williams J, Warnke R, Goffinet DR, Bagshaw MA. Carcinoma of the nasopharynx-the significance of histology. Int J Radiat Oncol Biol Phys. 1978; 4:199-205. [PubMed: 640890]

25. Reddy SP, Raslan WF, Gooneratne S, Kathuria S, Marks JE. Prognostic significance of keratinization in nasopharyngeal carcinoma. Am J Otolaryngol. 1995; 16:103-108. [PubMed: 7540805]

26. Marks JE, Phillips JL, Menck HR. The National Cancer Data Base report on the relationship of race and national origin to the histology of nasopharyngeal carcinoma. Cancer. 1998; 83:582-588. [PubMed: 9690553]

27. Polesel J, Franceschi S, Talamini R, et al. Tobacco smoking, alcohol drinking, and the risk of different histological types of nasopharyngeal cancer in a low-risk population. Oral Oncol. 2011; 47:541-545. [PubMed: 21478046]

28. Giovino GA. The tobacco epidemic in the United States. Am J Prev Med. 2007; 33(6 Suppl):S318S326. [PubMed: 18021906]

29. Tyan YS, Liu ST, Ong WR, Chen ML, Shu CH, Chang YS. Detection of Epstein-Barr virus and human papillomavirus in head and neck tumors. J Clin Microbiol. 1993; 31:53-56. [PubMed: 8380183] 
A

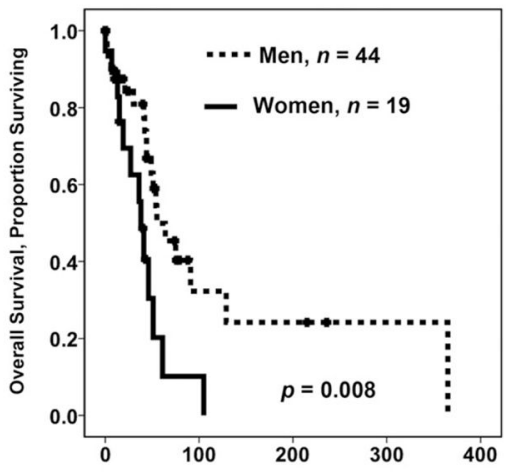

C

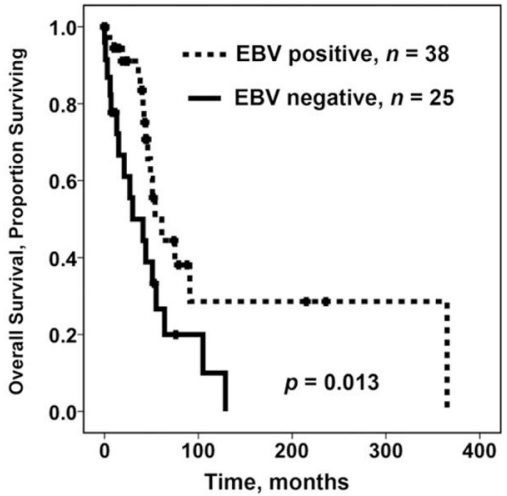

B

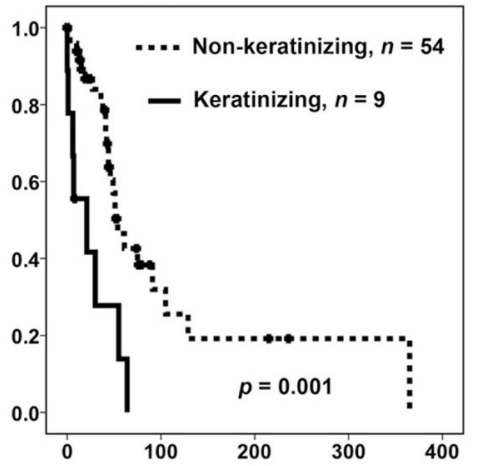

D

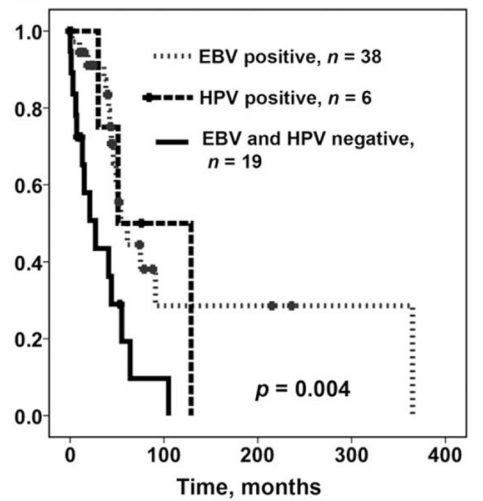

FIGURE 1.

Prognostic factors and overall survival (OS) of patients with nasopharyngeal carcinoma, univariate analysis. (A) Sex and OS. (B) Histology of nasopharyngeal carcinoma (keratinizing vs nonkeratinizing) and OS. (C) Nasopharyngeal carcinoma, Epstein-Barr virus (EBV) status, and OS. (D) Epstein-Barr virus and human papillomavirus status and OS. 


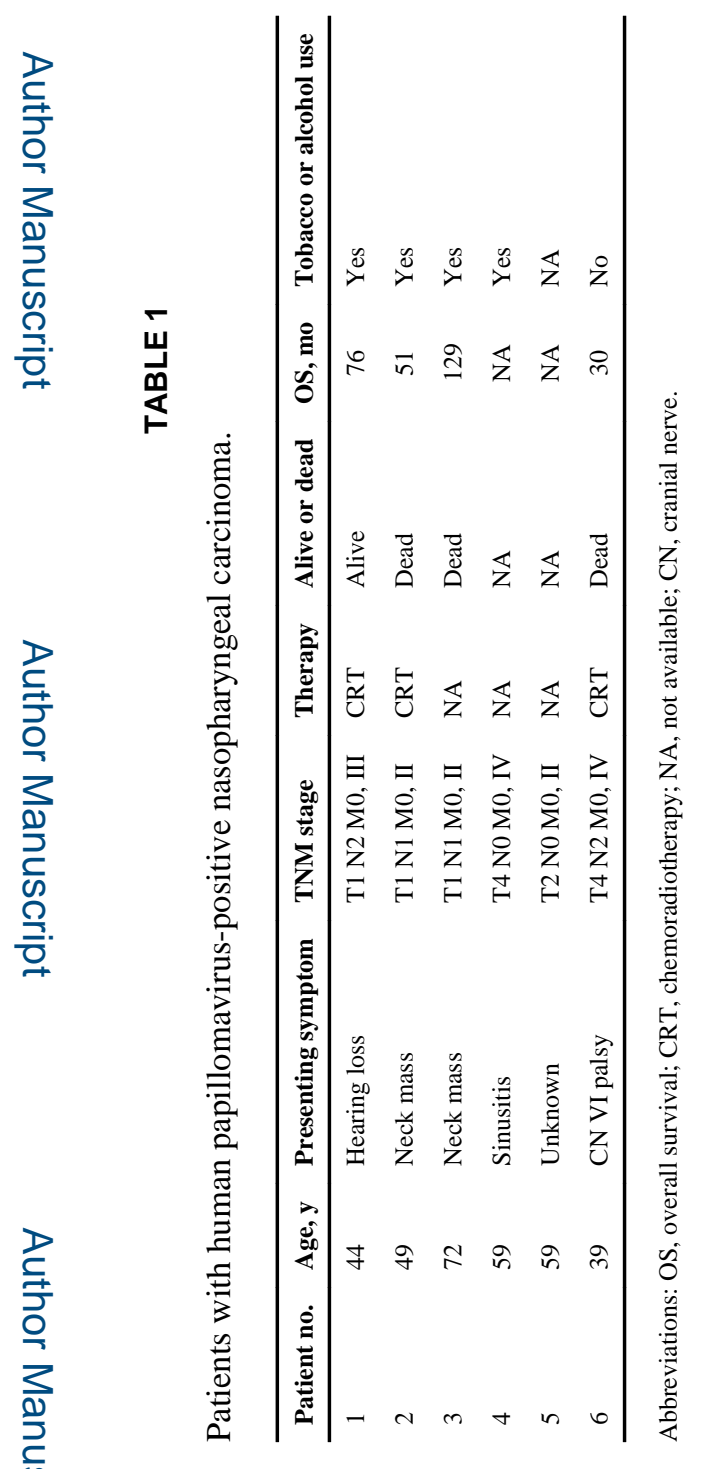


TABLE 2

Decrease in prevalence of keratinizing nasopharyngeal carcinoma, 1956 to 1971 and 1981 to 2012.

\begin{tabular}{|c|c|c|c|}
\hline Feature & Group 1, 1981 - March $2012(n=63)$ & Group 2, 1956-1977 $(n=29)$ & $p$ value \\
\hline \multicolumn{4}{|l|}{ Sex } \\
\hline Male & 44 & 25 & .09 \\
\hline Female & 19 & 4 & \\
\hline \multicolumn{4}{|l|}{ Histology } \\
\hline Keratinizing & 9 & 10 & .026 \\
\hline Nonkeratinizing & 54 & 19 & \\
\hline \multicolumn{4}{|c|}{ In situ hybridization and p16 IHC } \\
\hline EBV positive & 38 & $15^{*}$ & \\
\hline HPV/p16 positive & 6 & Not tested & \\
\hline EBV and HPV/p16 negative & 19 & EBV negative -10 & \\
\hline
\end{tabular}

Head Neck. Author manuscript; available in PMC 2015 November 23. 


\section{TABLE 3}

Clinicopathological features of patients with nasopharyngeal carcinoma, 1981 to 2012.

\begin{tabular}{|c|c|c|c|c|}
\hline & EBV-pos/unknown HPV* $(n=38)$ & EBV-neg/HPV-pos $(n=6)$ & EBV-neg/HPV-neg $(n=19)$ & $p$ value \\
\hline Average age, y. & 50.5 & 53.6 & 57.8 & $>.05$ \\
\hline Men/women & $29 / 9$ & $5 / 1$ & $10 / 9$ & $>.05$ \\
\hline \multicolumn{5}{|l|}{$\operatorname{Race}^{\dagger}$} \\
\hline White & 33 & 6 & 17 & $>.05$ \\
\hline African American & 2 & 0 & 2 & \\
\hline \multicolumn{5}{|l|}{ Smoking } \\
\hline Ever & 10 & 4 & 5 & $>.05$ \\
\hline Never & 8 & 1 & 3 & \\
\hline \multicolumn{5}{|l|}{$\mathrm{T}$ classification $\S$} \\
\hline 1 & 4 & 2 & 2 & $>.05$ \\
\hline 2 & 4 & 0 & 0 & \\
\hline 3 & 5 & 0 & 1 & \\
\hline 4 & 5 & 2 & 4 & \\
\hline \multicolumn{5}{|l|}{$\mathrm{N}$ classification $\|$} \\
\hline 0 & 1 & 0 & 4 & $>.05$ \\
\hline 1 & 8 & 2 & 5 & \\
\hline 2 & 8 & 2 & 3 & \\
\hline 3 & 5 & 0 & 0 & \\
\hline \multicolumn{5}{|l|}{ Clinical stage $\mathscr{I}$} \\
\hline II & 6 & 2 & 2 & $>.05$ \\
\hline III & 12 & 1 & 4 & \\
\hline IV & 8 & 2 & 5 & \\
\hline \multicolumn{5}{|l|}{ Hist. type } \\
\hline Ker. & 0 & 1 & 8 & $<.001$ \\
\hline NK & 38 & 5 & 11 & \\
\hline \multicolumn{5}{|l|}{ Therapy $^{* *}$} \\
\hline CRT & 29 & 3 & 7 & $>.05$ \\
\hline $\mathrm{RT}$ & 5 & 0 & 4 & \\
\hline Median follow-up, mo & 56 & 47.6 & 24.2 & .02 \\
\hline
\end{tabular}

Abbreviations: EBV, Epstein-Barr virus; Pos., positive; HPV, human papillomavirus; Neg., negative; Hist., histological type; Ker., keratinizing; NK, nonkeratinizing; CRT, chemoradiotherapy; RT, radiotherapy.

* Eight EBV-positive nasopharyngeal carcinoma (NPC) were shown to be HPV in situ hybridization (ISH) and p16 immunohistochemical (IHC)negative.

${ }^{\dagger}$ In the group of EBV-positive/unknown HPV nasopharyngeal carcinomas, 2 were of Asian race and 1 patient was of Middle Eastern ethnicity.

${ }^{+}$Smoking history was unknown for 32 patients (EBV-positive/unknown HPV, $n=20$; EBV-negative/HPV-positive, $n=1$; EBV-negative/HPVnegative, $n=11$ ).

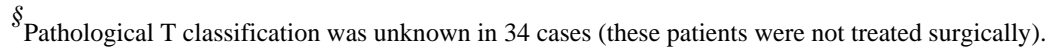

Head Neck. Author manuscript; available in PMC 2015 November 23. 


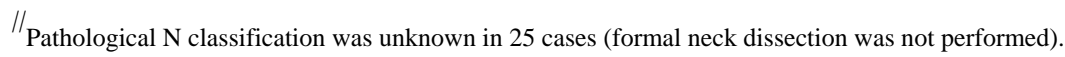

${ }^{\text {II }}$ Clinical stage was unknown for 19 patients. One EBV-negative/HPV-negative patient presented with stage I disease. **

Therapeutic modality was unknown for 13 patients and 2 patients received no treatment.

Therapeutic modity was unkown for 13 patients and 2 patiens received no treats. 